\title{
Invasive infections caused by Streptococcus pneumoniae in a tertiary-level children's hospital before the introduction of the conjugate vaccine. Clinical characteristics and serotypes involved
}

\author{
Guadalupe M. Pérez, M.D. ', Adriana Parra, M.D. ${ }^{a}$, Lidia Casimir, M.D. ${ }^{b}$, \\ Alejandra Mastroianni, Biochemist ${ }^{b}$, Vanesa Reijtman, Biochemist ${ }^{b}$, \\ Horacio Lopardo, M.D. ${ }^{b}$ and Rosa Bologna, M.D. ${ }^{a}$
}

\begin{abstract}
Invasive pneumococcal diseases are the main cause of morbidity and mortality in children. In the Hospital "Prof. Dr. Juan P. Garrahan", between October $1^{\text {st }}, 2008$ and September $30^{\text {th }}$, 2011 all invasive pneumococcal diseases with positive blood cultures were retrospectively studied before the implementation of the universal immunization schedule with the 13 -valent pneumococcal conjugate vaccine. A total of 124 patients were identified, and their mean age was 48.3 months (range: 1-216). In this population, $58.9 \%$ (n: 73 ) were OVER 2 years old and $89 \%$ (n: 65$)$ of them had an underlying disease. The most frequent clinical presentation was pneumonia. The most frequent S. pneumoniae serotypes identified were: $14(22.5 \%, \mathrm{n}: 25), 6$ (14.4\%, n: 16), 19 (8.1\%, n: 9), 23 (7.2\%, n: 8), 1 (6.3\%, n: 7$), 5(4.5 \%, \mathrm{n}: 5)$, and 7 (7.2\%, n: 8$)$. Of the $S$. pneumoniae serotypes in this series, $82.2 \%$ is included in the 13 -valent pneumococcal conjugate vaccine. Continuous epidemiological surveillance is essential to further identify the epidemiology and study the evolution of invasive pneumococcal disease in Argentina.

Key words: pneumococcal disease, Streptococcus pneumoniae, pneumococcal serotypes, pneumonia, bacteremia.
\end{abstract}

http:/ /dx.doi.org/10.5546/aap.2013.202

a. Department of Epidemiology and Infectious Disease Control.

b. Department of Microbiology.

Hospital Nacional de Pediatría "Prof. Dr.

Juan P. Garrahan".

E-mail adress:

Guadalupe M. Pérez,

M.D.: guaperez@

hotmail.com

Conflict of interest:

None

Received: 8-24-12

Accepted: 1-2-13 pneumococcal conjugate vaccine was added to the national immunization schedule. ${ }^{6}$ This study describes the clinical characteristics and serotypes involved in pneumococcal invasive diseases confirmed by blood cultures in Hospital de Pediatría "Prof. Dr. Juan P. Garrahan" over the three years previous to adding this vaccine to the immunization program.

\section{MATERIAL AND METHODS}

Invasive infections caused by S. pneumoniae were retrospectively studied from October 2008 to September 2011 in Hospital "Prof. Dr. Juan P. Garrahan", a children's tertiary care hospital with 620 beds and 4 intensive care units where children from all over the country are referred to. All children with a clinical condition compatible with S. pneumoniae and positive blood cultures recorded in the hospital during the study period were included. Patients with blood cultures tested at a different hospital were excluded.

Medical records were reviewed to document each patient's history as well as the characteristics of the clinical course and evolution. Results were submitted for publication after ensuring that patients' identity would be kept confidential. The study was conducted in accordance with the Declaration of Helsinki ethical principles. Serotyping of the 111 pneumococcal isolates available at the time of the study was performed in the Infectious Disease National 
Institute of the National Administration of Labs and Health Institutions (INEI-ANLIS) "Dr. Carlos G. Malbrán" using the Quellung reaction with the antisera provided by the Statens Serum Institut, Copenhagen, Denmark.

\section{RESULTS}

Between October 2008 and September 2011, 124 patients with blood cultures positive for $S$. pneumoniae and symptomatology compatible with invasive pneumococcal disease were identified. Out of them, $50.8 \%$ (n: 63) were male. The mean age was 48.3 months (range: 1-216). Of them, $67.7 \%$ (n: 84) had an underlying disease. Predominant comorbidities were: hematooncological disease (44.4\%, n: 36$)$, nephrotic syndrome (9.9\%, n: 8), immunodeficiencies (12.8\%, n: 10), and congenital heart defects $(6.2 \%, \mathrm{n}: 5)$. Only 10 patients $(8.1 \%)$ had received a pneumococcal vaccine. Mortality rate was $9.7 \%$ (n: 12 ).

Patients' characteristics were analyzed depending on whether they were older or younger than 2 years old (Table 1). Patients older than 2 years old accounted for $58.9 \%$ of the population (n: 73), and 89\% (n: 65) of them had an underlying disease. The presence of an underlying disease was predominant in the older than 2 year old group $(<0.01)$.

The most frequent presentation of the invasive disease caused by S. pneumoniae was pneumonia: $60.8 \%$ (n: 31 ) in patients younger than 2 years old and $49.3 \%$ (n: 36 ) in patients older than 2 years old. Considering both groups, $30 \%$ had sepsis. Primary peritonitis was present in $5.9 \%$ (n: 3 ) of the patients younger than 2 years old and in $13.7 \%$ (n: 10) of children older than 2 years old.
The most frequent serotypes of S. pneumoniae were: $14(22.5 \%, \mathrm{n}: 25), 6(14.4 \%, \mathrm{n}: 16)$, $19(8.1 \%, \mathrm{n}: 9), 23(7.2 \%, \mathrm{n:}), 1(6.3 \%, \mathrm{n}: 7), 5(4.5 \%$, n: 5), and 7 (7.2\%, n: 8) (Figure 1).

\section{DISCUSSION}

Invasive infection caused by S. pneumoniae is still the main cause of severe disease in children worldwide. ${ }^{1}$ It is the most common cause of community-acquired bacterial pneumonia among non-vaccinated populations ${ }^{7}$ and the etiologic agent of community-acquired sepsis and meningitis. In Argentina, population studies have estimated an annual incidence of invasive disease similar to that found in other countries before the implementation of the universal immunization schedule. $^{8}$

The incidence of invasive disease caused by S. pneumoniae, reported in the bibliography, is higher among patients under 2 years old and immunocompromised patients. ${ }^{1}$ However, in the series run at Hospital Garrahan, the incidence was higher in children older than 2 years old, a finding which is probably related to the characteristics of the hospital population (patients with chronic conditions, hematooncological disease, immunodeficiencies, and heart disease).

In this series, pneumonia, with or without pleural empyema, was the most predominant clinical presentation, followed by sepsis and fever without a source. This is consistent with the bibliography, which describes pneumonia, followed by occult bacteremia and sepsis, as prevalent syndromes. ${ }^{9}$ Given the association with the nephrotic syndrome and the characteristics of the underlying disease in the studied population,

TABLE 1. Demographic, clinical outcomes characteristics of the population by age

\begin{tabular}{|c|c|c|c|c|}
\hline Characteristic & Total & Younger than 2 years old & Older than 2 years old & $\mathrm{p}^{*}$ \\
\hline$\%(n)$ & $100(124)$ & $41.1(51)$ & $58.9(73)$ & NS \\
\hline Underlying disease \% (n) & $67.7(84)$ & $37.3(19)$ & $89(65)$ & $<0.01$ \\
\hline Male \% (n) & $50.8(63)$ & $51(26)$ & $50.7(37)$ & NS \\
\hline Mean age in months (range) & $48.3(1-216)$ & $10.7(1-22)$ & $74.6(24-216)$ & - \\
\hline \multicolumn{5}{|l|}{ Clinical presentation \% (n) } \\
\hline Fever without a source & $16.9(21)$ & $11.8(6)$ & $20.5(15)$ & NS \\
\hline Meningitis & $0.8(1)$ & - & $1.4(1)$ & NS \\
\hline Pneumonia (with or without effusion) & $54(67)$ & $60.8(31)$ & $49.3(36)$ & NS \\
\hline Focus Osteoarticular & $1,6(2)$ & $3,9(2)$ & - & - \\
\hline Peritonitis & $10.4(13)$ & $5.9(3)$ & $13.7(10)$ & NS \\
\hline Sepsis & $29.8(37)$ & $29.4(15)$ & $30.1(22)$ & NS \\
\hline Death & $9.6(12)$ & $9.8(5)$ & $9.6(7)$ & NS \\
\hline
\end{tabular}

${ }^{*} \chi 2$ with and without Yates' correction and Fisher's exact test. 
primary peritonitis was also found to be a frequent presentation in this series. Mortality in this study was higher than the rate described in specialized literature. ${ }^{10}$ It should be noted that $67.7 \%$ of children had associated comorbidities, which favor the occurrence of more serious infections.

The recent incorporation of the 13-valent pneumococcal conjugate vaccine for children younger than 2 years old in Argentina's immunization schedule is an effective strategy evaluated in other countries for the decrease of invasive disease $\mathrm{e}^{11}$ and nasopharyngeal carriage of S. pneumoniae. ${ }^{12}$

The 13-valent pneumococcal conjugate vaccine includes capsular antigens of serotypes 1, 3, 4, 5, $6 \mathrm{~A}, 6 \mathrm{~B}, 7 \mathrm{~F}, 9 \mathrm{~V}, 14,18 \mathrm{C}, 19 \mathrm{~A}, 19 \mathrm{~F}$ and $23 \mathrm{~F} .{ }^{5} \mathrm{O}$ the serotypes found in this series, $82.8 \%$ is included in the vaccine. If only children younger than 2 years old are studied, $85.6 \%$ of the serotypes identified in this study are covered by the 13-valent pneumococcal conjugate vaccine.

In countries where universal immunization has been performed with the 7-valent conjugate vaccine for years, non vaccine serotypes have emerged ${ }^{13,14}$ and the clinical characteristics of the invasive infection caused by S. pneumoniae have changed. ${ }^{15}$ In Argentina, universal immunization in children younger than 2 years old with the 13-valent pneumococcal conjugate vaccine has been in place since 2012. It is necessary to maintain a careful epidemiological surveillance and to continuously study incidence, evolution and clinical presentations of the invasive pneumococcal disease and its predominant serotypes.

\section{REFERENCES}

1. Paganini H. Infectología Pediátrica. 1ra edición. Editorial Científica Interamericana, 2007: 975-985.

2. Kaplan SL, Mason EO Jr, Wald ER Schutze GE et al. Decrease of invasive pneumococcal infections in children among 8 children's hospitals in the United States after the introduction of the 7-valent pneumococcal conjugate vaccine. Pediatrics 2004;113:443-49.

3. Ferro Bricks L,Berezin E. Impact of pneumococcal conjugate vaccine on the prevention of invasive pneumococcal diseases. J Pediatr (Rio J). 2006;82(3):S67-74.

4. PírezMC,Algorta G,Cedrés A,SobreroHetal.Impactodela vacunación neumocócica universal en las hospitalizaciones por neumonía y meningitis en niños de Montevideo Uruguay. Pediatr Infect Dis J 2011;8:669-74.

5. Lexau C, Lynfield R, Danila R, Pilishvili T et al. Changing epidemiology of invasive pneumococal disease among older adults in the era of pediatric pneumococcal conjugate vaccine. JAMA 2005;294:2043-51.

6. Ministerio de Salud de la Nación Argentina. Resolución 502/2011.

7. Bradley J, Byington C, Shah S, Alverson B et al. The management of community-acquired pneumonia in infants and children older than 3 months of age: Clinical Practice Guidelines by the Pediatric Infectious Diseases Society and the Infectious Diseases Society of America. Clin Infect Dis 2011;53:617-30.

8. Tregnaghi M, Ceballos A, Rüttimann R, Ussher J et al. Active epidemiologic surveillance of pneumonia and invasive pneumococcal disease in ambulatory and hospitalized infants in Cordoba, Argentina. Pediatr Infect Dis J 2006;25:370-2.

9. Klugman KP, Feldman C. Streptococcus pneumoniae respiratory tract infections. Curr Opin Infect Dis 2001;14:1739.

10. Christensen JS, Jensen TG, Kolmos HJ, Pedersen $C$ et al. Bacteremia with Streptococcus pneumoniae: sepsis and other risk factors for 30-day mortality-a hospital-based cohort study. Eur J Clin Microbiol Infect Dis 2012; 13 (epub ahead of print).

11. McIntoshEDGHow many episodes of hospital caremightbe prevented by widespread uptake of neumococcal conjugate vaccine?. Arch of Dis in Child 2003; 88:859-61.

12. Rinta-Kokko H,Dagan R,Givon-Lavi N,Auranen K. Estimation of vaccine efficacy against acquisition of pneumococcal carriage. Vaccine. 2009; 27:3831-7.

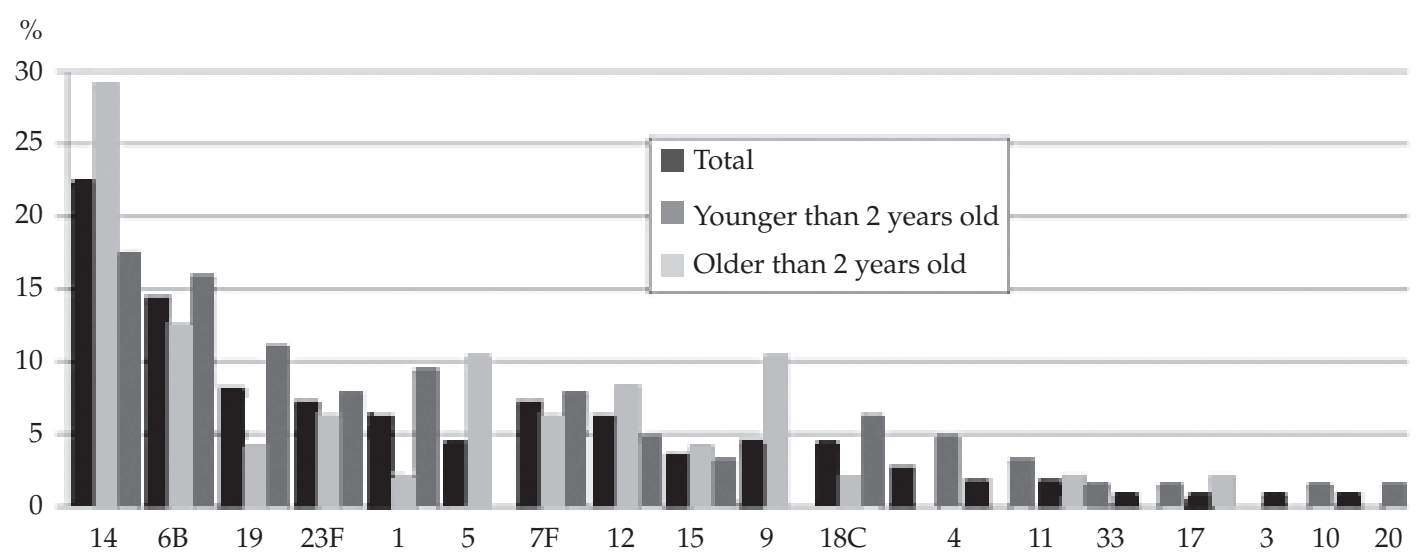


13. Kaplan S, Barson W, Lin P, Stovall S et al. Infections in children serotype 19A is the mostcommon serotype causing invasive pneumococcal. Pediatrics 2010;125:429-36.

14. de Sevilla MF, García-García JJ, Esteva C, Moraga F et al. Clinical presentation of invasive pneumococcal disease in Spain in the era of heptavalent conjugate vaccine. Pediatr Infect Dis J 2012;31:124-8.
15. Ampofo K, Pavia AT, Chris S, Hersh AL et al. The changing epidemiology of invasive pneumococcal disease at a tertiary children's hospital through the 7-valent pneumococcal conjugate vaccine era: a case for continuous surveillance. Pediatr Infect Dis J 2012;31:228-34. 